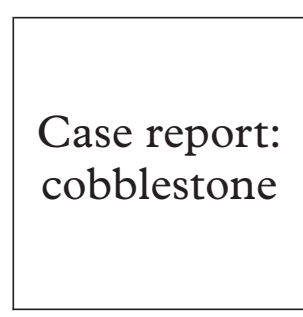

\title{
Neuropsychiatric reaction induced by clarithromycin in a patient on highly active antiretroviral therapy (HAART)
}

\author{
K Prime, P French
}

\section{Case history}

A 58 year old man, diagnosed HIV-1 antibody positive 3 years previously, presented with a 24 hour history of hyperactivity. This culminated in the development of suicidal and homicidal ideation. For fear of injury to himself and others he was admitted to hospital.

He had commenced a course of clarithromycin by mouth $500 \mathrm{mg}$ twice daily 10 days previously, for a bacterial chest infection. Two months before this, HAART had been commenced with zidovudine by mouth $250 \mathrm{mg}$ twice daily, didanosine by mouth $200 \mathrm{mg}$ twice daily, and nevirapine by mouth $200 \mathrm{mg}$ twice daily. This was in addition to long term treatment with simvastatin by mouth $20 \mathrm{mg}$ once daily for hypercholesterolaemia. There was no other medical history of note. He also had no previous history of mental ill health, alcohol, or drug misuse.

On physical examination, he was afebrile with oxygen saturations of $100 \%$ on air. His pulse was regular at 80 beats/minute and blood pressure 110/60 mm Hg. Examination of his respiratory and gastrointestinal systems was unremarkable. Neurological examination was unremarkable apart from mental state assessment which showed hyperactivity manifested as pressure of speech, poor concentration, and extreme anxiety. He also expressed suicidal and homicidal ideation into which he maintained insight.

Investigations including full blood count, urea and electrolytes, liver function tests, calcium, phosphate, and glucose were normal. Blood culture, Toxoplasma gondii serology, treponemal haemagglutination assay, and serum cryptococcal antigen were negative. HIV viral load was $<500$ copies/ml and CD 4 count $480 \times 10^{6} / 1$. Drug levels were not measured.

A diagnosis of a severe neuropsychiatric reaction secondary to clarithromycin was made. Clarithromycin was discontinued and he was treated with diazepam by mouth $5 \mathrm{mg}$ twice daily and temazepam by mouth $20 \mathrm{mg}$ at night. Twenty four hours after admission he remained only mildly hyperactive with no further suicidal or homicidal ideation. After 72 hours his symptoms had resolved completely and he was discharged.

\section{Discussion}

Clarithromycin, a macrolide antibiotic, and nevirapine, a non-nucleoside reverse transcriptase inhibitor, are principally metabolised

\section{Key messages}

- To raise awareness of the possibility of unexpected drug interactions between HAART and other more established drugs particularly with reference to cytochrome P450 induction/inhibition.

- To raise awareness of the CSM/MCA/ MRC blue card HIV drug toxicity reporting scheme.

- To be aware of drug interactions as a differential diagnosis in patients on HAART presenting with acute psychiatric syndromes.

in the liver by the cytochrome P450 isoenzymes of the CYP3A family. The major presystemic active metabolite of clarithromycin is the R-epimer of 14-OH-6-0-methylerythromycin A. Clarithromycin is an inhibitor of CYP3A and its use in patients concurrently taking drugs metabolised by the cytochrome P450 system may be associated with elevations in their serum levels. Nevirapine acts as a mild to moderate enzyme inducer of CYP3A. It may therefore not only reduce plasma concentrations of other drugs metabolised by the same system but also acts as an autoinducer, with steady state reached after 7-9 days.

The principal side effects of clarithromycin are gastrointestinal; however, less commonly, CNS side effects have been reported, including dizziness, vertigo, anxiety, insomnia, tinnitus, confusion, disorientation, hallucinations, psychosis, and depersonalisation. ${ }^{1}$ At the time of writing the Committee on Safety of Medicines (CSM)/Medicines Control Agency (MCA) have received 219 reports of suspected clarithromycin induced psychiatric disorders including psychosis $(n=5)$, depersonalisation $(n=5)$, and suicidal ideation $(n=4) \quad(C S M$, personal communication). It has been postulated that macrolide antibiotics may cause a psychosis-like syndrome via their inhibitory action on glutamatergic neurotransmission in the brain. ${ }^{2}$ Nevirapine has not been reported to cause serious CNS adverse effects. ${ }^{3}$

In one study $(n=18)$, concomitant administration of clarithromycin and nevirapine resulted in a $28 \%$ significant increase in nevirapine $\mathrm{C}_{\min }$ and a non-significant $26 \%$ increase in nevirapine area under the curve (AUC) and $\mathrm{C}_{\max }(24 \%)$, with no notable adverse effects. There was also a significant reduction in clarithromycin AUC (30\%) with a 58\% significant 
increase in AUC of its active 14-OH metabolite. ${ }^{3}$ A similar interaction study $(n=15)$ confirmed these results although AUC increase of the active metabolite $14-\mathrm{OH}$ clarithromycin was only $27 \%{ }^{4}$

To date, the CSM/MCA have received no reports of suspected interactions between either zidovudine, didanosine or simvastatin and clarithromycin or nevirapine.

We therefore postulate that the accumulation of the active 14-OH metabolite of clarithromycin caused the acute neuropsychiatric reaction observed in our patient. The mechanism for this reaction is not clear.

Clarithromycin is frequently used in the treatment of bacterial respiratory tract infections and disseminated Mycobacterium avium complex infection in HIV positive individuals. We wish to alert physicians to the potential for interactions between established drugs with well documented side effects, such as macrolide antibiotics, and the much newer classes of antiretroviral agents (ARVs) used in the treatment of HIV infection.

We thank Professor David Back and Sara Gibbons at the Department of Pharmacology and Therapeutics, University of Liverpool, for their helpful comments and advice regarding this paper; and the patient and staff on the Patrick Manson Unit for permission to report this case.

Competing interests: None declared.

1 Abbott Laboratories, 2000. Clarithromycin: summary of product characteristics. Available from the ABPI compendium of data sheets and SPCs 1999-2000.

2 Manev $\mathrm{H}$, Favaron M. Macrolide antibiotics protect neurons in culture against the N-methyl-D-aspartate receptor-mediated toxicity of glutamate. Brain Res 1993; 624:331-5.

3 Boehringer Ingelheim International, April 2000. Viramune: summary of product characteristics. Available from http:// www.eudra.org/humandocs/humans/EPAR/Viramune/ Viramune.htm

4 Robinson P, Gigliotti M. Effects of the reverse transcriptase inhibitor, nevirapine, on the steady-state pharmacokinetics of clarithromycin in HIV-positive patients. 6th Conference on Retroviruses and Opportunistic Infections, Chicago 1999. (Abstract 374.) 